\title{
Grammatiche lessicalmente esaustive delle passioni Il caso dell'Io collerico. Le forme nominali
}

\author{
Emilio D'Agostino
}

Università degli Studi di Salerno

\section{Abstract}

In base alle più comuni definizioni della parola passione, sembra delinearsi un quadro nel quale alcuni stati emotivi - alcuni relativamente stabili alcuni altri no - presentano fondamentalmente il carattere dell'eccesso e della sofferenza acuta. Delle passioni, più in particolare, sulla base della definizione del dizionario, appare dominante il carattere perturbante: la ragione, il giudizio, l'equilibrio, a loro contrapposti, sono condannati a subirne, a patirne, gli attacchi. L'universo del non-razionale, in tal senso, apparirebbe contraddistinto, non solo dall'assenza del logos, ma dalla presenza di slanci esplosivi volti alla negazione e alla distruzione di questo. Tra l'irrazionale, l'irragionevole e l'eccessivo costituirebbero una forza dirompente, difficilmente controllabile. Tali definizioni, in realtà, sembrano riprendere, semplificandola, la lunghissima e complessa tradizione che dall'epica omerica alle scienze cognitive contemporanee ha affrontato il tema delle passioni. Ad una di esse, quella primigenia dell'ira, è dedicato questo contributo di indagine lessico-sintattica.

Parole chiave: passione, ira, negazione, indagine lessico-sintattica.

\section{Abstract}

On the basis of most common definitions of the word passion, it seems to delineate a picture in which some emotional states present the character of excess and acute pain. According to dictionary definitions, perturbation is the dominant feature of passions: reason, sense, moderation are forced to suffer its attacks. The non-rational universe seems to be marked not only by the absence of logos but also by the presence of uncontrollable, disruptive explosive fits directed to the negation and destruction of logos. Dictionary definitions simplify the complex tradition that, from Homeric epics to Cognitive Sciences, has confronted the topic of passion. This paper is devoted to a lexical and syntactic analysis of one particular passion: ire.

Key words: passion, ire, negation, lexical and syntactic analysis. 
Solo la passione è il segno dell'esistenza

FEUERBACH, Grundsätze der Philosophie der Zukunft 26

\section{Premessa}

Se si esaminano le tre definizioni dei lemmi passione, emozione e sentimento in un comune dizionario d'uso dell'italiano, ad esempio il Grande Dizionario dell'italiano di De Mauro:

(1) Passione

1. OB LE patimento fisico: io veggo te star fermo nella tua acerba crudeltà né poterti la mia passione in parte alcuna muovere (Boccaccio)

2a. CO TS relig. spec. con iniz. maiusc., insieme dei tormenti e delle sofferenze sopportate sulla croce da Gesù Cristo | TS relig. narrazione evangelica di tali sofferenze: la P. secondo Luca

2b. BU fig., pop., sofferenza prolungata: la degenza in ospedale è stata una passione

3a. TS mus. grandiosa composizione musicale, nata nel secolo XVII, in origine polifonica e successivamente monodica e polifonica, che ha come tema il martirio e la morte di Cristo in croce

3b. TS lett., teatr. sacra rappresentazione che ha come tema il martirio e la morte di Gesù Cristo in croce

4. BU sofferenza morale, spirituale

5. FO sentimento che per le sue caratteristiche e la sua forza domina l'intera personalità, talvolta turbandone l'equilibrio psichico: non riesce a reprimere le sue passioni, la gelosia è una passione pericolosa

6. FO amore violento e caratterizzato da forte sensualità: una passione incontenibile, passione senile | estens., chi ha ispirato tale sentimento: è stato solo una passione, lei è stata la più grande passione della sua vita

7. FO forte interesse e inclinazione per qcs.: passione politica, passione per il calcio; persona dalle forti passioni: chi ha un temperamento fortemente passionale | l'oggetto che ispira tali sentimenti: la musica è la sua più grande passione

8. TS psic. tendenza dominante che svolge un'azione direttrice sul pensiero e sulla condotta, influenzando i giudizi di valore e modificando variamente i processi logici

9. CO parzialità, atteggiamento condizionato dai sentimenti e quindi privo di obiettività e razionalità: giudicare con passione, valutazione condizionata dalla passione

DATA: av. 1294.

ET Sinonimi: febbre, frenesia, fuoco, furore, tormento.

Contrari: apatia.

IMO: dal lat. passio ${ }^{n} e(m)$, der. di $p a \breve{t} t i$ «sopportare, ${ }^{1}$ patire».

(2) Emozione

1. FO impressione, sensazione forte: provocare, suscitare una forte emozione, un'emozione violenta; un viaggio ricco di emozioni, che emozione rivederti:; 
turbamento, agitazione: essere in preda all'emozione, non riuscire a parlare per l'emozione

2. TS psic. intensa esperienza psichica, piacevole o spiacevole, accompagnata da reazioni fisiche e comportamentali

Sinonimi: commozione, turbamento. DATA: 1644.

ETIMO: dal lat. tardo emotio ${ }^{-} n e(m)$, der. di emove ${ }^{-}$e «smuovere», cfr. fr. émotion.

(3) Sentimento

1. stato emotivo relativamente stabile derivato da un moto interiore di marca positiva o negativa, che si può o meno manifestare esteriormente: suscitare, ispirare un sentimento paterno, materno, essere spinti da un sentimento di pietà, provare un sentimento di gioia, nutrire sentimenti di odio, $i$ miei sentimenti verso di lei non cambieranno mai $\mid$ sensazione intermedia tra lo psichico e il fisico nata in seguito a un stimolo sensoriale o a un'elaborazione mentale: provo un sentimento di nausea per il degrado di questa città, provo un sentimento di disgusto per la sporcizia di questo quartiere

2. spec. sing., l'affettività in contrapposizione alla ragione e all'intelletto: lasciarsi guidare dal sentimento in una scelta, farsi travolgere dal sentimento

3a. sensibilità, finezza di sentire: una ragazza piena di sentimento

3b. slancio affettivo o partecipazione emotiva carica di calore e di intensità: abbracciare, baciare, parlare, suonare con sentimento

Sinonimi: anima.

4. spec. al pl., modo di pensare, sentire, comportarsi indicativo della morale e dell'etica individuale: buoni, onesti, bassi, ignobili sentimenti

5. coscienza, percezione di realtà di carattere spirituale, morale, civile, ecc., assunte come valori: il sentimento dell'onore, del perdono, del dovere, della fratellanza

6. CO fam., piena consapevolezza di sé e delle proprie azioni, senno: essere fuori, uscire di sentimento; con tutti i sentimenti, con ogni cura

Sinonimi: senno.

7. BU senso: $i$ cinque sentimenti; perdere i sentimenti, svenire; tornare in sentimenti, rinvenire

DATA: ca. 1300.

ETIMO: dal lat. mediev. sentime $\breve{u} t u(m)$, v. anche ${ }^{1}$ sentire,

si noteranno alcuni tratti definizionali fondamentali. Per il primo, la Passione: il patimento forte, intenso, prolungato, la sofferenza, capace di turbare l'equilibrio, di influenzare i giudizi di valore e di modificare i processi logici, di determinare la parzialità del comportamento e del giudizio, un sentimento privo di obbiettività e razionalità, suoi sinonimi sono febbre, frenesia, fuoco, furore, tormento, suo contrario apatia, suo dominio più tipico quello amoroso, suo esempio più frequente il martirio e la passione del Cristo. Per il secondo, l'Emozione: la sensazione forte, l'intesa esperienza psichica, la capacità di turbare l'animo. Per il terzo, il Sentimento: uno stato emotivo relativamente stabile, uno slancio, contrapposto alla ragione e all'intelletto, può essere, però, usato anche come sinonimo di senno. 
Riguardando le tre definizioni nel loro complesso, sembra delinearsi un quadro nel quale, tra gli stati emotivi, relativamente stabili alcuni altri no, alcuni tra essi presentano fondamentalmente il carattere dell'eccesso e della sofferenza acuta. Delle passioni, più in particolare, sulla base della definizione del dizionario, appare dominante il carattere perturbante: la ragione, il giudizio, l'equilibrio, a loro contrapposti, sono condannati a subirne, a patirne, gli attacchi. L'universo del non-razionale, in tal senso, apparirebbe contraddistinto, non solo dall'assenza del logos, ma dalla presenza di slanci esplosivi volti alla negazione e alla distruzione di questo. Tra l'irrazionale, l'irragionevole e l'eccessivo costituirebbero una forza dirompente, difficilmente controllabile. In modo schematico:

Ragione vs. Emozione

Passione $=$ emozione eccessiva

Tali definizioni, in realtà, sembrano riprendere, semplificandola, la lunghissima e complessa tradizione che dall'epica omerica alle scienze cognitive contemporanee ha affrontato il tema delle passioni. In esse, è riflessa, però, una parte soltanto di tale tradizione, quello che potremmo dire escludente. L'altra, quella che potremmo dire includente è taciuta. Le passioni rappresentano modi di essere e di sentire di tipo relazionale, attraverso le quali ci si colloca in rapporto con le persone, le situazioni e i valori storicamente dati della nostra collettività. Costituiscono una reazione forte e radicale, talvolta estrema, dissonante rispetto alla cosiddetta "normalità», in quanto in ogni passione c'è quasi sempre una dimensione anomala e trasgressiva rispetto alle consuetudini del nostro contesto, che, pertanto, finisce con il giudicare chi la manifesta come un essere dominato dagli istinti. In realtà, passioni ed emozioni giocano un ruolo fondamentale nell'attività cognitiva degli individui e il rapporto con la ragione è stato negli ultimi decenni, in vari ambiti di ricerca, riesaminato, eliminando i poli estremi della contrapposizione. Non è vero che la passione sia totalmente priva di rapporto con una ragione profonda. C’è sempre una ragione dentro la passione. L'altro estremo contestato oggi è che la passione possa rientrare tutta in una prospettiva razionale. La passione, da questo punto di vista, rappresenta un momento di fortissimo sentire, legato ad una ulteriore componente razionale, un progetto, un senso, che l'individuo vuole attribuire ad una relazione con l'oggetto con cui si relaziona. ${ }^{1}$

In questo contributo, che segue quello sul vocabolario della "menzogna», il tema affrontato è quello dell'espressione linguistica delle passioni, e mi occuperò di un "apparato significante», per dirla con Moravia (1995), che nasce

1. Su tali temi rimando a A. R. DAMASIO, Descartes' Error. Emotion, Reason and the Human Brain, trad. it. L'errore di Cartesio. Emozione, ragione e cervello umano, Milano: Adelphi, 1995 e The Feeling of What Happens. Body and Emotion in the Making of Consciousness, trad. it. Emozione e coscienza, Milano: Adelphi, 2000; D. GOLEMAN, Emotional Intelligence, trad. it. Intelligenza emotiva, Milano: Mondadori, 1996. 
con l'inizio stesso della letteratura universale e che manifesta il concetto, per l'appunto, di Passione. Non affronterò tanto il tema delle parole sulla passione quanto, piuttosto, il tema delle parole della passione: non di tutte, ammesso che sia possibile riprodurre oggi una nuova nosografia delle passioni come quella stoica, ma di un dominio particolare, quello dell'ira. Ad altri domini come, ad esempio, quello del desiderio dedicherò un altro contributo. Nel paragrafo successivo, tenterò di giustificare il perché di tale scelta, per il momento credo di poter dire soltanto che l'Ira è associata alla prima apparizione e definizione della soggettività occidentale, nel mondo omerico, e che di lì, attraverso una storia complicata è giunta sino a noi.

L'approccio qui seguito, quindi, sarà di tipo strettamente linguistico, perché mi occuperò delle parole che una lingua naturale offre all' uso comune (le parole della passione), non del lessico specializzato (le parole sulla passione), anche se non sarà possibile ignorare del tutto quella lunga storia ambivalente del concetto di Passione. ${ }^{2}$

\section{L'Ira: un'emozione fondamentale}

E' possibile rintracciare l'ira (o il suo più immediato sinonimo rabbia) nella manualistica della psicologia delle emozioni. Ad esempio in D'Urso-Trentin ${ }^{3}$ si legge come la rabbia (o ira) sia un'emozione tipica considerata fondamentale e inclusa tra $\mathrm{i}$ "sette vizi capitali» anche se, diversamente dall'odio che è definito un tipico stato di lunga durata, l'ira presenta il carattere di un'accensione momentanea. Essa costituisce una fra le più precoci tra le emozioni insieme alla gioia e al dolore, ed è considerata come «la tipica reazione alla frustrazione e alla costrizione sia fisica che psicologica» e insieme al disgusto e al disprezzo fa parte della cosiddetta "triade dell'ostilità». Per quanto sia sottoposta ad una forte pressione sociale negativa, essa colpisce individui di ogni classe ed età. All'ira si associa una tipica espressione facciale riconosciuta in tutte le culture studiate: l'aggrottare violento delle sopracciglia e lo scoprire e il digrignare i denti, oppure lo stringere fortemente le labbra, mentre il corpo tende fin quasi all'immobilità per poi accentuare notevolmente l'attività motoria. Ad un «volto dell'ira» si accompagnano anche condizioni fisiche particolari come l'aumento del battito cardiaco e la respirazione accelerata ed irregolare. Le sensazioni soggettive più comuni sono calore, irrigidimento della muscolatura, irrequietezza continua, paura di perdere il controllo, la voce spesso si alza di volume e di intensità, il tono può essere minaccioso, stridulo o sibilante. Essa è considerata alla stregua di uno stato emotivo capace di creare nell'organismo «un propellente energetico» utilizzabile per l'azione o per l'espressione verbale. Una delle cause principali della rabbia è rappresentata dalle minacce all'auto-stima o dai tentativi da parte di altri di imporre la loro volontà. D'Urso ${ }^{4}$ disegna

2. In ogni caso rimando a E. D'Agostino, Le parole dell'ira. Lessico e grammatica, in stampa.

3. Introduzione alla psicologia delle emozioni, Bari: Laterza, 1998.

4. V. D’Urso, Arrabbiarsi, Bologna: il Mulino, 2001. 
anche un quadro sintetico dell'immagine dell'arrabbiato nel quale quasi ognuno può facilmente riconoscersi anche nelle modalità linguistico-comunicative: uso del tu, frasi tronche, espressioni dialettali, coprolaliche, a sfondo sessuale, uso di forme imperative ecc. Anche se probabilmente è tanto diffusa tra gli uomini come tra le donne, diverso è il grado di accettazione sociale rispettivo, infatti il giudizio negativo è più forte nei confronti delle seconde. Trentin-D'Urso notano come vi siano molti termini della lingua che fanno riferimento a questa reazione emotiva. Infatti, ira, collera e rabbia sono considerati, in italiano, sinonimi di uno stato emotivo intenso, mentre se ne registrano altri che descrivono lo stesso sentimento ma in intensità minore (irritazione, fastidio, corruccio, impazienza) e anche qualcuno che, invece, ne accentua l'intensità (esasperazione, furore), oppure altri come accanimento che ne indicano il protrarsi. Si può notare ancora con gli autori, come in italiano sembrino dominanti la localizzazione e le conseguenze della rabbia nel corpo (non ci vide più dalla rabbia, si rodeva il fegato), l'espressione linguistica di una dimensione di passività e di patologia (è stato più forte di me, perdere le staffe, pazzo di rabbia) e la convinzione che il controllo della rabbia faccia male alla salute fisica ancor più che a quella mentale (se non glielo dicevo sarei scoppiato, era così controllato che gli è venuto l'ulcera, la cirrosi). ${ }^{5}$

Anche nel più recente studio di Canali-Pani si sottolinea il carattere fondamentale di tale emozione, "madre» di tutte le altre ed il legame con l'aggressività di specie:

L'ira è una delle emozioni basilari, forse la madre di tutte le emozioni, impossibile dire di aver vissuto senza averla mai provata, difficile definirla un vizio, a meno che non si intenda come vizio quello di non essere riusciti a controllarla e di essere, quindi, vittime della carne che è in noi, dell'animale che l'uomo era, è ancora e troppo spesso dimentica di essere. Per parlare dell'ira e della rabbia, dei gesti irrazionali e dell'aggressività da un punto di vista biologico, bisogna tutto sommato parlare di emotività in generale. Proprio così, il vizio è tutto qui, è solo il difficile controllo delle proprie emozioni. Scusate se vi sembra poco. $^{6}$ (p. 244)

L'ira, in tal senso, parente stretta dell'aggressività, rappresenta il primo buon esempio di Passione che, in quanto tale, allontana dalla Ragione, poiché chi ne è colto perde la padronanza delle proprie azioni, quasi fosse sotto l'azione di un agente esterno, come fanno immaginare espressioni come essere assalito dall'ira, tenere l'ira sotto controllo, lottare contro l'ira. D'altronde, in questa stessa condizione sembrano trovarsi tutte le passioni in generale e la loro complessa «nebulosa».

5. Cfr. V. D’urso, R. TREnTIN, Introduzione alla psicologia delle emozioni, Bari: Laterza, 1998, p. 312.

6. S. CANALI, L. PANI, Emozioni e malattia. Dall'evoluzione biologica al tramanto del pensiero psicosomatico, Milano: Mondadori, 2003. 
D'Urso, ${ }^{7}$ riprendendo Averill, ${ }^{8}$ ricorda come un'emozione come l'ira possa essere interpretata, nella prospettiva della costruzione sociale delle emozioni, anche come "ruolo sociale transitorio», con il quale impersoniamo l'arrabbiato secondo il copione che la società suggerisce, con le sue norme esplicite, ma soprattutto quelle implicite, modellando il nostro modo di sentire. D'altro canto, Anolli, a proposito del cosiddetto "lessico emotivo" sottolinea come esista una grande differenza tra la semantica e l'estensione dei singoli repertori emotivi:

Si va dal lessico emotivo inglese che contiene oltre 2000 parole a quello olandese con 1500 termini per giungere al lessico cinese (di Taiwan) con 750 parole e alla lingua di Malay con 230 termini. Fra le culture preletterate gli Ifaluk hanno un vocabolario emotivo composto di 58 termini, mentre i Chewong fruiscono soltanto di una decina di parole per esprimere tutte le emozioni. ${ }^{9}$

E' interessante notare, inoltre, come non sussista una corrispondenza per la medesima categoria emotiva fra una lingua e l'altra. In particolare, Anolli cita il caso proprio della collera (p. 112):

per esempio, la categoria collera in italiano non corrisponde al liget degli ilongor (una tribù dei cacciatori di teste nelle Filippine centrali) che, assieme alla collera, comprende anche la fierezza della competizione, la baldanza e il bisogno di provare se stessi. Né corrisponde al song degli ifaluk della Micronesia, per i quali il song è un insieme di collera, riprovazione, di rifiuto del cibo e autopunizione per punire l'altro. A sua volta, la categoria di ikari della lingua giapponese soltanto in parte equivale a collera, in quanto, pur esprimendo astio verso l'avversario, comporta una tendenza ad allontarsi, un sentimento di impotenza e di inibizione.

Per quanto riguarda le opinioni sull'ira, in lingue come l'italiano, D'Urso ${ }^{10}$ riporta una serie di espressioni linguistiche comuni che vanno dal riferimento mitologico (essere un'Erinni), all'energia (incanalare la rabbia accumulata, a furor di popolo), agli eventi metereologici (la sua faccia si era rannuvolata, è di umore burrascoso) alla dinamica dei gas (comprimere la propria rabbia), al fuoco (attizzare la rabbia, dare in escandenze), alla crescita graduale (ribollire d'ira, la sua rabbia trabboccò), all'attivazione brusca (sbottare, avere uno scatto d'ira), alla descrizione delle manifestazioni e delle conseguenze (stare con la bava alla bocca, fremere di rabbia, digrignare i denti, vedere rosso, scoppiare le vene, mangiarsi il fegato dalla rabbia, farsi il sangue amaro, andare in bestia, essere inferocito, infierire, urla belluine, essere una belva, essere inviperito, perdere il lume della ragione, essere fuori di sé), alle espressioni idiomatiche (perdere le staf-

7. Op. cit.

8. J. R. AVERILl, Anger and Aggression: An Essay on Emotion, New York: Springer Verlag, 1982.

9. L. Anolli (a cura di), Psicologia della comunicazione, Bologna: il Mulino, 2002, p. 111.

10. Op. cit. 
$f e$, perdere i freni, girare i coglioni, farsi venire $i$ cinque minuti, uscire dai gangheri, alzarsi col piede sbagliato, avere un diavolo per capello).

\section{L'ira di Achille}

Achille, figlio di Peleo, re di Ftia in Tessaglia, e della dea Teti figlia di Oceano: l'oggetto del racconto de I'Iliade è per l'appunto la sua collera. Della causa della famosa «debolezza» del suo tallone sono narrate due leggende. La prima vuole che, immerso nel fuoco dalla madre per eliminare la sua parte mortale, si bruciasse il labbro e il soprosso del piede destro. Curato dal centauro Chirone con l'osso di un gigante famoso per le sue doti di corridore, deve a quest'episodio la sua fama di uomo veloce. La seconda vuole che, immerso nello Stige dalla madre per assicurargli l'immortalità, solo sempre il famoso tallone, per il quale lei lo sosteneva, rimanesse mortale. Partecipò alla guerra di Troia alla testa dei Mirmidoni e prese parte anche all'operazione su Lirnesso nella quale, mentre Agamennone prendeva Criseide, lui fece altrettanto con Briseide: le due donne origine della disputa tra i due re nel decimo anno dell'assedio. E' con questa disputa che ha inizio propriamente la narrazione omerica. Malgrado alcuni tratti gentili, la figura di Achille è stata tramandata dai filosofi greci, specie gli Stoici, come uomo violento, schiavo delle passioni e proprio per queste ragioni lo si oppone normalmente ad Ulisse.

E’ F. Codino che, nell'introduzione alla traduzione de L'Iliade, ricorda come, contrariamente a quanto accadeva nella poesia eroica di tradizione orale, l'ira dell'eroe semidio occupi lo spazio di tutto il poema:

Nell'Iliade non si parla solo dell'ira di Achille; anzi in molti libri non se ne parla affatto. Ma è notevole che essa sia messa talmente in evidenza fino dalla prima parola che potrebbe dare il titolo al poema e che attorno ad essa sia costruita un'opera così ampia. (p. XII)

D'altronde, non soltanto è Achille ad essere dominato dall'ira, ma ad esserlo sono molti degli eroi rappresentati nel poema, tranne Ulisse. Ad esempio, lo stesso Agamennone nelle scene iniziali del Libro I:

Così detto, sedette; s'alzò fra loro

l'eroe figlio d'Atreo, il molto potente Agamennone, infuriato; d'ira tremendamente i neri precordi,

lo stesso Ettore, nel libro IX, è rappresentato dagli ambasciatori presso la tenda di Achille nel modo seguente:

... Ettore, ebbro della sua forza,

infuria spaventoso, ché sfida Zeus, e non rispetta

uomini o déi, lo ha preso una rabbia selvaggia.

erano gonfi, gli occhi parevano fuoco lampeggiante 
E', però, comunque Achille a dominare il palcoscenico dell'eccesso:

\section{Libro I}

Canta, o dea, l'ira d'Achille Pelide,

rovinosa, che infiniti dolori inflisse agli Achei,

gettò in preda all'Ade molte vite gagliarde

d'eroi, ne fece il bottino dei cani,

di tutti gli uccelli - consiglio di Zeus si compiva -

da quando prima si divisero contendendo

l'Atride signore d'eroi e Achille glorioso.

...

Disse così; al Pelide venne dolore,

il suo cuore nel petto peloso fu incerto fra due:

se, sfilando la daga acuta via dalla coscia,

facesse alzare gli altri, ammazzasse l'Atride,

o se calmasse l'ira e contenesse il cuore.

E gli parlò la dea Atena occhio azzurro:

Io venni a calmar la tua ira ... da come:

Nel saggio sulla sacralizzazione della violenza e del sangue, Ehrenreich ricor-

il momento forse più triste di tutto il cruento poema è la trasformazione di Achille da bellissimo eroe in belva sanguinaria e cannibalica. Al termine del loro duello, Achille chiama Ettore morente «carne» e si rammarica che il proprio appetito non sia più forte. ${ }^{11}$

Infatti, Ettore è per Achille krea:

\section{Libro XXII}

No, cane, non mi pregare, né pei ginocchi né pei genitori;

ah! Che la rabbia e il furore dovrebbero spingere me

a tagliuzzar le tue carni e a divorarle così, per quel che mi hai fatto.

Achille, se in battaglia è contrapposto ad Ettore, nel proprio campo trova il suo controaltare, come s'è detto, in Ulisse che in primo luogo è descritto saggio e abile con le parole:

\section{Libro II}

Disse così, non disobbedì la dea Atena occhio azzurro

e si lanciò d'un balzo giù dalle vette d'Olimpo.

Giunse rapidamente alle agili navi degli Achei;

trovò Odisseo, simile a Zeus per saggezza

11. B. Ehrenreich, Blood rites. Origins and history of the passions of war, New York: H. Holt Co., 1997, p. 53, trad. it. Riti di sangue. All'origine della passione della guerra, Milano: Feltrinelli, 1998. 
Figlio glorioso di Laerte, abile Odisseo

con le tue blande parole trattieni ognuno degli uomini

ma, che per la sua metis, rientra nella nostra mitologia come «l'ingannatore» e «il mentitore» per eccellenza, il «polipo» opposto alla «dritta volpe», il motivo decorativo sinuoso a ellissi messo contro quello più arcaico lineare di cui parla Tagliapietra: ${ }^{12}$

\section{Libro III}

Quello è il figlio di Laerte, Odisseo abilissimo, 200 che crebbe tra il popolo d'Itaca, la ricca di rocce, e conosce ogni sorta d'inganni e di acuti pensieri

Con Vegetti ${ }^{13}$ va notato come LIliade si apra con la parola menis, la famosa ira di Achille, ma come, allo stesso tempo, Omero utilizzi ben quattro termini per indicare ciò che parzialmente si traduce con l'italiano ira. Vegetti nota come, in realtà, per descrivere la «nebulosa collerica» si alternino le seguenti parole:

menis «'indignazione» «il risentimento violento»

cholos «la collera aspra ed amara»

menos «il furore guerriero in campo di battaglia»

thimòs «'impulso emotivo che scatena l'azione»

e a proposito dell'emozione provata da Achille nota (p. 40):

la prima e ancora incerta percezione di sé come soggetto sia pur precariamente unificato di azione avviene dunque nel fuoco dell'emozione collerica, nella reazione violenta ed aggressiva alla minaccia che viene dall'altro. La fragilità antropologica della figura del signore eroico rende questa minaccia mortale, come nel caso di Achille. Ogni lesione alla sua dignità e al suo onore (timé) che si produca nelle dinamiche di interazione sociale viene avvertita come catastrofica, perché la signoria dell'eroe, il suo comando sul gruppo umano che gli è sottoposto, non hanno alcuna forma di legittimazione se non la continua e tenace riaffermazione del suo valore e quindi del suo potere. [...] Nello spazio dell'ira che separa l'offesa dalla vendetta si produce dunque la prima autoconfigurazione di un soggetto che più tardi sarebbe stato chiamato "passionale», ma che ora è «eroico» e non conosce alternative possibili.

Ma se la menis penetra anche Ettore l'illustre, il luminoso e il più amato dagli déi a Ilio, si impossessa di lui, come forza passionale quasi esterna al suo cuore, come nella similitudine del Libro XXII:

12. A. Tagliapietra, Filosofia della bugia. Figure della menzogna nella storia del pensiero occidentale, Milano: Mondadori, 2001.

13. S. Vegetti Finzi (a cura di), Storia delle passioni, Bari: Laterza, 1995. 
Come serpente montano attende l'uomo sopra il suo buco,

mali veleni ha mangiato, lo penetra collera atroce, guarda fisso, terribile, arrotolato sopra il suo buco; così Ettore, con inestinguibile ardore, non arretrava, ma poggiando lo scudo lucente al gradino del muro, diceva irato al suo cuore magnanimo...

Achille mostra nella rappresentazione omerica un tratto che è per certi versi sorprendente. Il riferimento è al Libro IX e all'ambasceria di Fenice, Aiace e Odisseo. Agammenone li ha inviati con promesse di doni per risarcire e quindi convincere Achille a tornare a combattere:

Ma poi che ho sbagliato seguendo pensieri funesti, io voglio soddisfarlo, dargli compenso infinito; e dirò a tutti voi i magnifici doni

Il re si esprime in termini di doron. I magnifici doni devono servire a porre rimedio all'ira e all'abbandono del pelide dopo che il suo geras, il bottino, gli è stato tolto dallo stesso Agamennone. Benveniste ${ }^{14}$ (1962) ricorda alcuni aspetti linguistici che a tale riguardo sono significativi. Il greco ha varie forme che si riferiscono tutte al "dono»: dós, dôron, doreá, dósis e dotíne, tutte con alla base la radice ${ }^{*} d o-$. In particolare, dôron e doreá sembrano avere lo stesso senso: il primo è il dono materiale, il secondo il fatto di destinare o portare questo. Dotíne, pur traducibile con dono, costituisce il termine più particolarizzato:

\section{Libro IX}

che l'onoreranno (timésousi) come un dio con offerte (dotínai)

ricchi tributi (liparàs thémistas) a lui pagheranno, sotto il suo scettro

Timésousi e thémistas, legati a timé, si associano alla nozione di "prerogativa del capo» e di «rispetto». Agamennone, dunque, riconosce il proprio errore e cerca di porvi rimedio, ma Achile, come è noto, rifiuta. La risposta ai tre ambasciatori che elencano i dora suona:

Ai capi, a re, donò premi d'onore,

che a loro restano intatti; solo a me fra gli Achei

l'ha ritolto, si tiene la sposa mia dolce.

Ma il premio, colui che l'ha dato

Se l'è ripreso, violento

14. E. BenVEnISTE, Le vocabulaire des institutions indo-européennes, Paris: Ed. Le Minuit, 1969, trad. it. Il vocabolario delle istituzioni indoeuropee, Torino: Einaudi, 1976. 
Mi sono odiosi i suoi doni, lo stimo quanto un capello.

Anche se dieci, venti volte di più mi donasse

Di quanto ora possiede...

Entrambi, così facendo, hanno infranto l'obbligo del dare-ricevere, come noterebbe Mauss riguardo al potlàc melanesiano e, quindi, non essendoci stato scambio, le loro anime, attraverso i doni, non si sono confuse. ${ }^{15}$ I tre emissari di Agamennone tacciono:

Parlò così: muti rimasero tutti, in silenzio,

scossi dalla parola; rifiutava con molta violenza

Fenice:

così sappiamo che anche il fiore degli uomini antichi,

degli eroi, se mai qualcuno vinceva l'ira violenta,

eran sensibili ai doni, aperti alle parole

Ma perché Achille rifiuta ed il suo risentimento prevale? Come mai non c'è riconciliazione possibile, nonostante, come nota Fenice, ciò sia sempre accaduto anche con gli déi mediante sacrifici e doni e, come suggerisce Aiace, anche nel caso dell'assassinio di un fratello o di un figlio? E' qui dunque, in questo scenario, che è possibile notare come appare un Io non più eteroprodotto, ma generato dalla coscienza di un Sé unitario e (almeno in parte) autonomo rispetto agli stessi déi. P. K. Feyerabend, nel suo ultimo lavoro pubblicato postumo, nota come Achille e i suoi interlocutori parlino due linguaggi diversi:

Muti rimangono tutti, in silenzio scossi dalla parola

e, se il linguaggio è una "costrizione contro l'esperienza» nel senso che è un tentativo collettivo di semplificare e sistemare l'esperienza in scatole chiuse ben maneggevoli, ${ }^{16}$ pur parlando Achille e i suoi interlocutori della stessa idea, l'onore, mentre per questi ultimi il comportamento di Agamennone è pienamente tollerabile, per il primo:

il merito è stato trascurato non solo nel suo caso, ma anche in altri, l'onore è orfano e l'ingiustizia da lui notata sta nella natura delle cose. ${ }^{17}$

Agamennone, intollerabilmente, è stato il primo a sottrarsi all'obbligo di «donare molto e dappertutto» (Mauss). Per l'epistemologo scomparso, Achil-

15. Cfr. M. Mauss, Essai sur le don, Paris: P.U.F., 1950, trad. it. Saggio sul dono. Forma e motivo dello scambio nelle società arcaiche, Torino: Einaudi, 1965 (in particolare il cap. II).

16. Feyerabend cita il saggio di Baxandall su Giotto (M. BAXANDALL, Giotto e gli umanisti, Milano: Jaca Book, 1994).

17. Cfr. P. K. Feyerabend, Conquest of Abundance, University of Cicago Press, 1999, p. 44 (trad. it. Conquista dell'abbondanza. Storie dello scontro fra astrazione e ricchezza dell'Essere, R. CorTina ed., Milano, 2002). 
le ha fatto una scoperta e, in ciò, la soggettività ha certamente svolto un ruolo, poichè è stata la sua ira ad agire da cassa di risonanza, facendogli notare ciò che gli altri non ancora avevano notato. Essersi dovuto privare di Briseide è per Achille, dunque, una ferita non rimarginabile, come dice Citati ${ }^{18}$ (2002):

una lacerazione che lo colpisce nell'intimo, nella ragione di essere e di vivere [...] Se questa è la prima volta che l'Io prorompe nella letteratura occidentale, si tratta di un'esplosione spaventosa, capace di distruggere il mondo. [...] L'offesa che ha ricevuto è inespiabile: non può venire riparata con doni, scambi di cose, o possessi. Egli non tollera che le passioni del suo io, la sua menis, vengano scambiate con oggetti.

\section{Ancora Feyerabend nota:}

Achille ha fatto una scoperta. La soggettività ha certamente svolto un ruolo: è stata l'ira di Achille ad agire da cassa di risonanza, facendogli notare ciò che gli altri non avevano ancora notato. ${ }^{19}$

E' dunque, molto verosimilmente, con l'Ira che inizia la storia del Soggetto nella cultura occidentale ed è la tradizione omerica che sta all'origine di tutte le rappresentazioni che di quella sono state date. Come, d'altronde, è sempre in quest'epica che va, molto probabilmente, rintracciata anche la nascita di un Desiderio non più anch'esso etero-prodotto, attraverso la raffigurazione in particolare del desiderio femminile. Come nota Sissa, ${ }^{20}$ Penelope non è soltanto "un'eroina del ricordo", come direbbe Citati, ma piuttosto la donna che, incerta e perplessa, dopo il sogno dell'aquila-Ulisse che uccide le sue ocheProci nel suo cortile (Odissea, Libro XIX), si appresta a scegliere un nuovo marito, non più sulla base dei ricchi doni promessi, ma sulla base di una spinta di puro carattere soggettivo e sensuale: una fisicità pari almeno a quella di Ulisse. Salvo il carattere delle virilocalità attraverso Telemaco, se Penelope è oggetto di desiderio in quanto donna, il desiderio in lei sarà destato nuovamente soltanto attraverso la fisicità della prova dell'arco. La sintesi dei caratteri dei due Soggetti, quello tragico e quello desiderante, la si ritroverà successivamente nell'appassionata e furiosa Medea di Euripide che coniugherà entrambe le dimensioni.

Nel tracciare una sorta di fortuna dell'ira non è possibile dimenticare le tradizioni legate alla filosofia greca (Platone e Aristotele), la tradizione veterotestamentaria e quella medievale — l'ira è uno dei sette peccati capitali - ma per ragioni di spazio si rimanda a D’Agostino (2004). 


\section{L'Ira nell'italiano contemporaneo}

Nei dizionari di uso comune dell'italiano si ritrovano definizioni come le seguenti:

Zanichelli

Ira

s. f.

1. collera, corruccio, furia, furore, indignazione, iracondia, irritazione, esacerbazione, rabbia, sdegno, stizza, bile, fiele, accanimento, arrabbiatura, isterismo CONTR. calma, placidità, quiete, tranquillità, mansuetudine, mitezza, flemma, impassibilità, imperturbabilità

2. (spec. al pl.) gravi discordie

3. (fig.) (di vento, di mare, ecc.) furia, veemenza

FRAS. è un'ira di Dio (fig., fam.), è una persona terribile, è una cosa terribile. $\mathrm{V}$. anche stizza

L'impeto dell'animo improvviso e violento che si rivolge contro qualcuno o qualcosa è denominato ira: infiammarsi, accendersi, avvampare, ardere d'ira; trattenere, placare l'ira; l'espressione essere accecato dall'ira rappresenta figuratamente l'essere oltremodo irato; una persona o cosa terribile e pericolosa si definisce invece nel linguaggio familiare con la locuzione è un’ira di Dio. Nell'ambito particolare della teologia cattolica l'ira è uno dei sette vizi capitali e consiste nell'ingiusto e smodato desiderio di vendetta. Chi per tendenza naturale o abituale è facile preda di questo sentimento si dice caratterizzato da irascibilità, ossia da iracondia. I sinonimi che più si avvicinano a ira sono collera, rabbia e arrabbiatura: andare, montare in collera; essere in collera con qualcuno; parole piene di rabbia; ho fatto prendere a mio padre una bella arrabbiatura. L'irritazione è invece lo stato o la condizione di chi ha perso la pazienza senza arrivare però agli accessi violenti che contraddistinguono l'ira e i suoi sinonimi precedenti: provo irritazione verso la sua ipocrisia. In particolare, un'irritazione acuta ma di breve durata, dovuta specialmente a scontentezza, contrarietà o impazienza, si dice stizza: reagire con un moto di stizza; questo termine corrisponde a bile usato in senso figurato: sputare, ingoiare bile; rodersi dalla bile; molto vicino è anche fiele, che però designa un'irritazione accompagnata da rancore, amarezza e astio: parole di fiele; essere pieno di fiele.

All'origine di questi moti dell'animo c'è di solito un sentimento di vivo risentimento o di riprovazione provocato da chi o da ciò che sembra intollerabile, ossia un sentimento di sdegno o indignazione: trattenere lo sdegno; muovere, suscitare la pubblica indignazione; per indicare uno sdegno misto a dolore $\mathrm{e}$ delusione si adopera il termine corruccio: dimostrare il proprio corruccio; sentire corruccio. Quando la reazione emotiva è sproporzionata a quanto l'ha provocata e sfocia in atti d'ira smodati e incontrollati si ricorre ai termini isteria ed isterismo, usati in senso estensivo e non specialistico e connotati naturalmente da una sfumatura negativa; l'accanimento è invece un odio tenace, una rabbia ostinata e quasi persecutoria contro qualcuno: perseguitare con accanimento un rivale. Ancora più intensi sono il furore e la furia, che consistono in una veemente agitazione collerica, per lo più di durata limitata, così violenta che quasi offusca la ragione: accendere qualcuno di furore; un furore momentaneo; placare il furore; lasciamogli sbollire la furia; andare su tutte le furie; entrambi questi termini sono equivalenti al significato figurato di ira, che indica lo sca- 
tenarsi degli elementi naturali: l'ira del mare; la furia del vento e della pioggia ha causato danni enormi; il furore delle acque distrusse il villaggio.

De Mauro

Ira

s.f.

1a. AU stato di violenta irritazione che tende a manifestarsi con parole di sdegno e gesti di collera aggressiva, indignazione e sim.: ira implacabile, feroce; scatto, sfogo d'ira; accendersi, avvampare, fremere d'ira; provocare, suscitare l'ira di qcn.; frenare, trattenere l'ira; sfogare la propria ira su qcn. | essere accecato dallira, essere adirato tanto da perdere il controllo di se stesso

Sinonimi: bile, collera, irritazione, rabbia, stizza.

1b. TS teol. uno dei sette peccati capitali che consiste in un violento e smodato desiderio di vendetta

2a. CO odio, risentimento: attirare su di sé le ire di qcn. | avere qcn. in ira, odiarlo, detestarlo | essere in ira a qcn., essere odiato o malvisto da qcn. Sinonimi: risentimento.

2b. BU spec. al pl., grave discordia: le ire cittadine, le ire di parte

3. LE giusto sdegno, nobile furore: nutrì contro a' Persi in Maratona... la virtù greca e l'ira (Foscolo)

Sinonimi: indignazione.

4. CO fig., violenza degli elementi naturali: ira del vento, del mare in tempesta

5. CO chi è fuori di sé per la rabbia, furia: sembrare unïra scatenata

In realtà, anche se Dante ha condannato ad essere straziato dalle altre anime Filippo Argenti nelle acque della palude Stigia (Canto VIII dell' Inferno) e Ludovico Ariosto all'inizio del suo poema cita l'ira e i giovenil furori d'Agramante, l'italiano contemporaneo usa ben poco i lemmi della «nebulosa collerica». Se si esaminano i dizionari di frequenza dello scritto (Lessico di frequenza dell'italiano contemporaneo) e del parlato (Lessico di frequenza dell'italiano parlato), si osserva come con i principali lemmi di tale dominio di significato e cioè collera, furia, furore, ira, rabbia e sdegno si registri un quadro come il seguente:

\begin{tabular}{ccc} 
scritto & & parlato \\
\hline- & collera & - \\
\hline+ & furia & + \\
\hline- & furore & - \\
\hline+ & ira & + \\
\hline+ & rabbia & - \\
\hline- & sdegno & - \\
\hline
\end{tabular}

con tre lemmi per lo scritto e soltanto due per il parlato. ${ }^{21} \mathrm{Se}$, si ricerca la forma volgare incazzarsi ci si accorge che, assente nello scritto, nel parlato ha il rango

21. In realtà, va notato come nel caso di furia possa aver influito nel calcolo, deviandolo, una forma come a furia di. 
3184 superiore a quello di ira (3818) e a quello di furia (4024). Tipica, quindi, del parlato dell'italiano contemporaneo, tale forma sembra occupare il ruolo principale come espressione generica dello stato emotivo in questione. ${ }^{22}$

\section{Lessico e Grammatica dell'Ira}

S’è detto all'inizio che questo era un contributo di ordine strettamente linguistico e descrittivo. Rimandando a Gross ${ }^{23}$ (1975) e, per l'italiano, ai normalmente citati EMDA (1981) e D’Agostino (1992), in quest'occasione è presentata una tavola lessico-grammaticali dei nomi dell'ira, costruita su un vocabolario di 170 lemmi. ${ }^{24}$

\subsection{I nomi dell'ira}

Le forme nominali incluse nella classificazione corrispondono tutte a forme predicative, come si rivela dalla correlazione con predicati verbali e/o con predicati aggettivali:

arrabbiatura - arrabbiarsi - arrabbiato

ira-irarsi-iroso

e dall'esistenza di un GN frutto di una nominalizzazione:

l'ira di Luca

lo sdegno di Ugo

la rabbia di Eva

e, per tale ragione, le forme di frase in questione sono state analizzate tutte in costruzioni a verbo supporto.

22. Per quanto concerne la sintassi delle forme composte legate al nome cazzo in italiano contemporaneo, si veda Elia (2002).

23. M. Gross, Méthodes en syntaxe, Paris: Hermann 1975.

24. adirarsi adiratamente adirato arrabbiamento arrabbiare arrabbiarsi arrabbiata arrabbiatamente arrabbiato arrabbiatura collera collericamente collerico incollerire incollerirsi incollerito contrariare contrariarsi contrariato contrarietà corrucciamento corrucciare corrucciarsi corrucciatamente corrucciato corruccio corrucciosamente corruccioso furente furentemente furia furibondamente furibondo furiosamente furioso furore imbufalire imbufalirsi imbufalito incacchiarsi incacchiato incacchiatura incavolarsi incavolato incavolatura incazzarsi incazzato incazzatura incazzosamente incazzoso incollerire incolle rirsi incollerito infuriare infuriarsi infuriatamente infuriato inquietarsi inquietato ira iracondamente iracondia iracondo iracundia iracundo irarsi irascersi irascersi irascibile irascibilità irascibilmente iratamene irato irosamente iroso irritabile irritabilità irritamento irritante irritare irritarsi irritatamente irritativo irritato irritatore irritazione rabbia rincollerire rincollerirsi rincollerito rinfuriare rinfuriato sdegnamento sdegnare sdegnarsi sdegnatamente sdegnato sdegnazione sdegno sdegnosaggine sdegnosamente sdegnosità sdegnoso stizza stizzare stizzarsi stizzire stizzirsi stizzito stizzosamente stizzoso. 


\section{$\mathrm{N} \mid \mathrm{N}$ \\ $\mathrm{O} \mathrm{O}$}

u

$=\mathrm{m}$

C o

h b

e b

1

F

-+ arrabbiamento

-+ arrabbiata

-+ arrabbiatura

-+ collera

-+ contrarietà

- + corrucciamento

-+ corruccio

-+ furia

-+ furore

-+ incacchiatura

-+ incavolatura

-+ incazzatura \begin{tabular}{|l|l|l|l|l|l|l|l|l|l|l|l|l}
$\mathrm{N}$ & $\mathrm{V}$ & $\mathrm{V}$ & $\mathrm{V}$ & $\mathrm{V}$ & $\mathrm{V}$ & $\mathrm{V}$ & $\mathrm{V}$ & $\mathrm{N}$ & $\mathrm{V}$ & $\mathrm{N}$ & $\mathrm{N}$ & $\mathrm{N}$
\end{tabular}

$\begin{array}{lllllllllllllllllll}\mathrm{O} & \mathrm{s} & \mathrm{s} & \mathrm{s} & \mathrm{S} & \mathrm{s} & \mathrm{s} & \mathrm{s} & \mathrm{O} & \mathrm{s} & \mathrm{O} & \mathrm{O} & \mathrm{O}\end{array}$ $\begin{array}{lllllllllllll} & u & u & u & U & u & u & u & & u\end{array}$

\begin{tabular}{ll|l|l|l|l|l|l|l|l|lllll}
$\mathrm{V}$ & $\mathrm{p}$ & $\mathrm{p}$ & $\mathrm{p}$ & $\mathrm{P}$ & $\mathrm{p}$ & $\mathrm{p}$ & $\mathrm{p}$ & $\mathrm{V}$ & $\mathrm{p}$ & $\mathrm{E}$ & $\mathrm{A}$ & $\mathrm{P}$
\end{tabular}

S

\begin{tabular}{llllllll|l|l|l|l|l|l}
$s$ & A & S & P & A & N & P & C & s & S & s & d & o
\end{tabular}

$\begin{array}{llllllllllllllllll}\text { u } & \text { v } & \text { e } & \text { r } & \text { V } & \text { u } & \text { r } & \text { o } & \text { u } & \text { o } & \text { e } & \text { a } & \text { v }\end{array}$

$\begin{array}{lllllllllllllllll}\mathrm{p} & \mathrm{e} & \mathrm{n} & \mathrm{o} & \mathrm{V} & \mathrm{t} & \mathrm{e} & \mathrm{v} & \mathrm{p} & \mathrm{f} & \mathrm{r} & \mathrm{r} & \mathrm{a}\end{array}$

$\begin{array}{lllllllllllllllllllll}r & t & \mathrm{v} & \mathrm{E} & \mathrm{r} & \mathrm{n} & \mathrm{a} & & \mathrm{f} & \mathrm{e} & \mathrm{e} & \mathrm{r}\end{array}$

$\begin{array}{llllllllllllllllllllll}\mathrm{N} & \text { e } & \text { i } & \text { a } & \text { R } & \text { i } & \text { d } & \text { r } & \text { d } & \text { r } & & & \text { e }\end{array}$ \begin{tabular}{l|l|l|l|l|l|l|l|l|l}
$r$ & r & T & r & e & e & i & i & i & i
\end{tabular}

\begin{tabular}{ll|l|l|l|l|l|l|l|l|l} 
e & e & I & e & r & & & r & n & n & u
\end{tabular}

\begin{tabular}{ll|l|l|l|l|l|l|l}
$\mathrm{R}$ & $\mathrm{s}$ & & $\mathrm{N}$ & $\mathrm{e}$ & & & $\mathrm{n}$ \\
$\mathrm{E}$ & $\mathrm{i}$ & & & & $\mathrm{N}$ & $\mathrm{N}$ &
\end{tabular}

S

e

$\mathrm{n}$

s

o

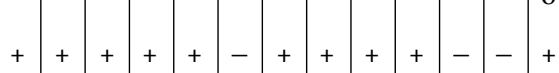

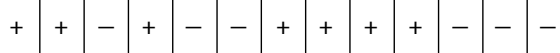

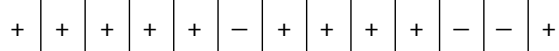

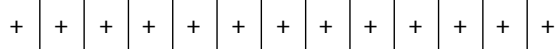

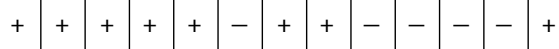

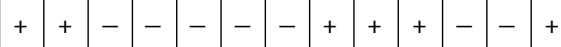

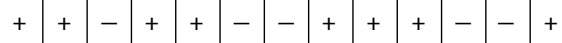

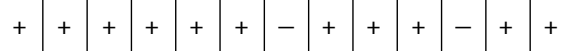

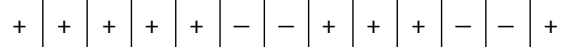

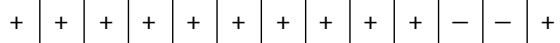

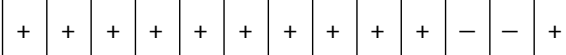

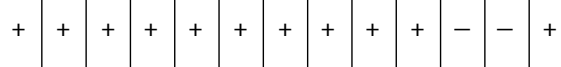




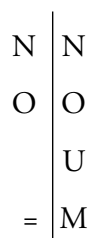

$\mathrm{C} \mathrm{O}$

h $\mathrm{B}$

e $\mathrm{B}$

$\mathrm{L}$

F

-+ ira

-+ iracondia

-+ iracundia

-+ irritamento

-+ irritazione

-+ rabbia

-+ sdegnamento

-+ sdegnazione

-+ sdegno

-+ sdegnosaggine

- + sdegnosità

-+ stizza

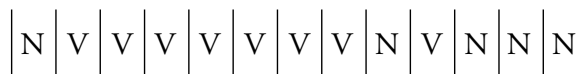

$\begin{array}{llllllllllllllll}\mathrm{O} & s & s & s & \mathrm{~S} & \mathrm{~s} & \mathrm{~s} & \mathrm{~s} & \mathrm{O} & \mathrm{s} & \mathrm{O} & \mathrm{O} & \mathrm{O}\end{array}$ $\begin{array}{llllllllll}u & u & u & U & u & u & u & & u\end{array}$ $\begin{array}{llllllllllllllllll}V & \text { p } & \text { p } & \text { p } & \text { P } & \text { p } & \text { p } & \text { p } & \text { V } & \text { p } & \text { E } & \text { A } & \text { P }\end{array}$ $S$ $\begin{array}{llllllllllllllll}\text { s } & \text { A } & \text { S } & \text { P } & \text { A } & \text { N } & \text { P } & \text { C } & \text { s } & \text { S } & \text { s } & \text { d } & \text { o }\end{array}$ $\begin{array}{llllllllllllllll}\text { u } & \text { v } & \text { e } & \text { r } & \text { V } & \text { u } & \text { r } & \text { o } & \text { u } & \text { o } & \text { e } & \text { a } & \text { v }\end{array}$ $\begin{array}{llllllllllllll}\text { p } & \text { e } & \text { n } & \text { o } & \text { V } & \text { t } & \text { e } & \text { v } & \text { p } & \text { f } & \text { r } & \text { r } & \text { a }\end{array}$

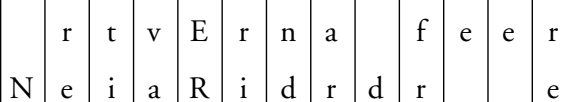
\begin{tabular}{|l|l|l|l|l|l|l|l|l|l|l|l|l}
$N$ & r & r & T & r & e & e & i & i & i & i & \\
& e & e & I & e & r & & & r & n & n & u \\
& & & R & & s & & & N & e & & & n \\
& & & & & & & & & & & &
\end{tabular} s e n $S$

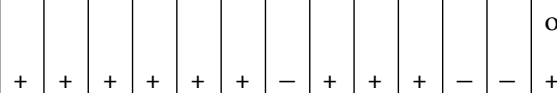

$+++++\infty-\infty+++-\infty$

$+++++C_{+}-++++-$

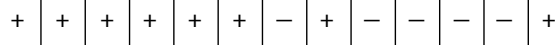

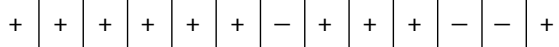

$+++++++-+++--_{-}+$

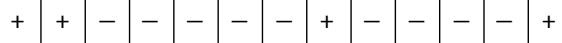

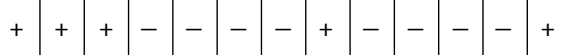

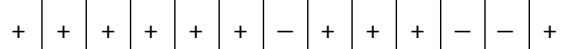

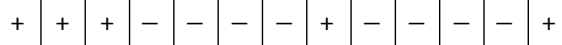

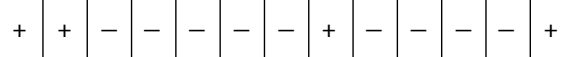

+ + + + + + + $+-|+|+|+|-\mid$ 
Sono state, dunque, esaminate le seguenti proprietà: proprietà distribuzionali e proprietà trasformazionali.

Nel caso delle proprietà distribuzionali, è stata presa in esame la posizione soggetto $\left(N_{0}\right)$. Esso non è mai un soggetto frastico $\left(N_{o}=:\right.$ Che $\left.F\right)$ e, per tale ragione, tali predicati nominali costituiscono una sotto-classe degli operatori elementari di tipo harrisiano, in particolare, della forma $\mathrm{O}(\mathrm{nn})$ :

\section{Eva si è presa un'arrabbiatura con Luca}

Ugo è in collera con Max

Luca prova sdegno nei confronti di Eva

Per quanto concerne, invece, le proprietà trasformazionali, sono state testate proprietà relative ai $V s u p$, in particolare, di alcuni di essi: avere, sentire, avvertire, nutrire, prendersi, covare, essere in, andare in.

Avere costituisce il supporto di uso generalizzato e correlato con Essere nella costruzione aggettivale equivalente:

Ugo ha rabbia nei confronti di Eva

Ugo è rabbioso nei confronti di Eva

Luca ha una forte collera per il tuo comportamento

Luca è fortemente incollerito per il tuo comportamento

e si conferma il loro comune statuto di verbi di «stato», come sostenuto in Benveniste $^{25}$ (1966):

Ugo ha uno stato d'ira

Ugo è in uno stato d'ira

costruzioni per le quali sussiste la relazione di coreferenza tra $\mathrm{N}_{0}$ e stato che dà luogo al GN:

\section{lo stato d'ira di Ugo}

Va notato, inoltre, che i GN del tipo Nira di $N_{o}$ possono rientrare in forme di frase «standard-incrociate» ${ }^{26}$ del tipo:

l'ira brillava negli occhi di Ugo

gli occhi di Ugo brillavano d'ira

Va notato, inoltre, come alla forma di frase con avere si correlano nella maggioranza dei casi le due costruzione con i supporti provare e sentire:

25. E. Benveniste, Etre et avoir dans leur fonctions linguistiques, in Problèmes de linguistique générale, Paris: Gallimard, 1966, vol. I.

26. Per la definizione di costruzione «standard-incrociata», si rimanda a A. EliA, M. MARTINELLI, E. D'AGostino, Lessico e strutture sintattiche. Introduzione alla sintassi del verbo, Napoli: Liguori, 1981; e a E. D’Agostino Analisi del discorso. Metodi descrittivi dellitaliano d'uso, Napoli: Loffredo, 1992. 


\section{Luca ha una forte collera nei confronti di Eva}

Luca prova una forte collera nei confronti di Eva

Luca sente una forte collera nei confronti di Eva

In tal senso, si nota, quindi, una «rete di supporti» ${ }^{27}$ costituita, da un lato, da essere, nelle costruzioni aggettivali, e, dall'altro, da avere e dalle possibili estensioni di questo come provare e sentire. Quindi, nel caso della presenza anche di una forma verbale correlata morfo-fonologicamente, si assisterà ad una classe di equivalenze come la seguente:

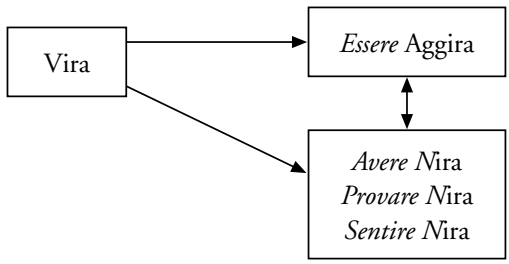

Con i due supporti provare e essere si rintracciano anche costruzioni del tipo:

provare un senso di $N=$ :

Luca ha provato un forte senso di sdegno nei confronti di Eva

essere in $N=$ :

Ugo è in collera con Luca

Nel primo caso, con senso in equivalenza con sentimento, ci si collega alla classe nominale delle «impressioni e modificazioni fisiche e psichiche ad opera di stimoli esterni o interni»:

Luca prova un senso di stanchezza (fisica)

* Luca prova un sentimento di stanchezza (fisica)

Luca prova un senso di (rabbia + depressione + angoscia)

Luca prova un sentimento di (rabbia + depressione + angoscia $)$

Nel secondo, invece, ci si collega a membri della classe di costruzioni di tipo "psicologico»:

Luca è in (angoscia + ansia $+{ }^{*}$ timore $+{ }^{*}$ paura $)$ per Eva

Infine, sono state esaminate estensioni come nutrire, prendersi, andare in e, caso a parte, l'estensione aspettuale covare: 
Eva nutre sdegno verso Luca

Ugo si è preso una forte arrabbiatura con Eva

Luca è andato in collera con Max

Max ha covato l'ira verso Eva a lungo

In realtà, l'interesse nei confronti dei nomi dell' ira è legato al fatto che essi rappresentano una sottoclasse ben caratterizzata di quelli che Gross ${ }^{28}$ definisce expressions de sentiment. In particolare essi, come già s'è detto, non accettano completive e infinitive in posizione soggetto, come invece accade con i nomi e i verbi che tradizionalmente sono stati chiamati "psicologici» che possono essere interpretati come causativi:
Eva è in angoscia
Eva è in collera
il tuo comportamento fa angosciare Eva
il tuo comportamento fa incollerire Eva
il tuo comportamento angoscia Eva
*il tuo comportamento incollerisce Eva ${ }^{29}$
il tuo comportamento (crea + causa) angoscia in Eva
il tuo comportamento (crea + causa) collera in Eva
il tuo comportamento è la causa dell'angoscia di Eva
il tuo comportamento è la causa della collera di Eva

e, pertanto, pur potendo naturalmente essere inserire in costruzioni causative non presentano intrinsecamente il tratto della causatività.

\section{Conclusioni}

In quest'occasione è stata presentata una classificazione di nomi legati al campo semantico dei sentimenti, in particolare quelli dell'ira. Se si confronta l'analisi proposta con quella avanzata precedentemente sui verbi della menzogna, si registrerà la differenza fondamentale per la quale se questi ultimi costituiscono una classe di operatori non elementari, quelli analizzati adesso, invece, rappresentano una classe di operatori elementari. In un'altra prospettiva, va evidenziato come l'italiano contemporaneo abbia "svuotato" tali forme di valori fortemente definizionali dell'individuo e si limiti, nell'uso comune, ad impiegare forme generiche e non caratterizzate.

28. M. Gross, «Une grammaire locale de l'expression de sentiment», in Langue française, 105, Paris: Larousse, 1995, p. 70-87.

29. In realtà, in questo caso è il pfx di derivazione in - a presentare un valore causativo. 